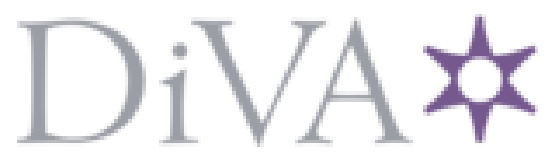

http://www.diva-portal.org

This is the published version of a paper published in Physical Review A. Atomic, Molecular, and Optical Physics.

Citation for the original published paper (version of record):

Linusson, P., Takahashi, O., Ueda, K., Eland, J., Feifel, R. (2011)

Structure sensitivity of double inner-shell holes in sulfur-containing molecules.

Physical Review A. Atomic, Molecular, and Optical Physics, 83(2): 022506

http://dx.doi.org/10.1103/PhysRevA.83.022506

Access to the published version may require subscription.

N.B. When citing this work, cite the original published paper.

Permanent link to this version:

http://urn.kb.se/resolve?urn=urn:nbn:se:uu:diva- 149572 


\title{
Structure sensitivity of double inner-shell holes in sulfur-containing molecules
}

\author{
P. Linusson, ${ }^{1}$ O. Takahashi, ${ }^{2}$ K. Ueda,${ }^{3}$ J. H. D. Eland,,${ }^{4,5}$ and R. Feifel ${ }^{5}$ \\ ${ }^{1}$ Department of Physics, Stockholm University, AlbaNova University Center, SE-106 91 Stockholm, Sweden \\ ${ }^{2}$ Department of Chemistry, Hiroshima University, Higashi-Hiroshima 739-8526, Japan \\ ${ }^{3}$ Institute of Multidisciplinary Research for Advanced Materials, Tohoku University, Sendai 980-8577, Japan \\ ${ }^{4}$ Department of Chemistry, Physical and Theoretical Chemistry Laboratory, Oxford University, \\ South Parks Road, Oxford OX1 3QZ, United Kingdom \\ ${ }^{5}$ Department of Physics and Astronomy, Uppsala University, Box 516, SE-751 20 Uppsala, Sweden
}

(Received 2 August 2010; published 18 February 2011)

\begin{abstract}
To demonstrate the structure sensitivity of double inner-shell hole spectroscopy, we have measured energies of $\mathrm{H}_{2} \mathrm{~S}^{2+}, \mathrm{SO}_{2}{ }^{2+}$, and $\mathrm{CS}_{2}{ }^{2+}$ with the two vacancies in the sulfur $2 p$ shell using a multielectron coincidence technique combined with synchrotron radiation. We describe how to extract intrinsic chemical information which is masked by the orbital relaxation effect in conventional core-level photoelectron spectroscopy.
\end{abstract}

DOI: 10.1103/PhysRevA.83.022506

PACS number(s): 33.70.Ca, 33.80.Eh, 34.50.Gb

\section{INTRODUCTION}

Photoelectron spectroscopy is known to reveal important insights into the electronic structure of matter. In particular, the relation of the binding energy of the core electron to the chemical environment, also known as the chemical shift, as accessible by core-level photoelectron spectroscopy [1], is used frequently in today's materials research. However, the experimentally observable chemical shifts of the single core-hole (SCH) states are not always sensitive to the chemical environment, as pointed out more than two decades ago by Cederbaum et al. [2].

In contrast to $\mathrm{SCH}$ creation, double core-hole $(\mathrm{DCH})$ creation is theoretically predicted to probe the chemical environment more sensitively [2-9]. Because of low ionization cross-sections, experimental investigations of DCHs have been extremely difficult until very recently. To study such states, it is of great advantage to have access either to a highly intense free electron laser (FEL) in the x-ray spectral region, as demonstrated in very recent works [10-12] performed at the Linac Coherent Light Source (LCLS) [13], or to a sensitive multielectron coincidence spectroscopy technique [14] using synchrotron radiation (SR). Using single-photon excitation for the DCH formation by SR, as done in Ref. [14] and in the present work, one can access primarily single-site DCHs, where the two inner-shell vacancies are situated on the same atomic site. By contrast, with two-photon excitation, not only single-site DCH states but also two-site DCH states, where the two inner-shell holes are at different atomic sites, are accessible [7-9,12]. It is theoretically well-established that energies of two-site DCH states exhibit more informative chemical shifts than the corresponding SCH states while those of single-site DCH states exhibit much larger orbital relaxation effects than the corresponding SCH states [2-9].

In order to demonstrate the power of DCH spectroscopy for chemical analysis, we present in this work a systematic investigation of single-site DCH states of a series of sulfurcontaining molecules: $\mathrm{H}_{2} \mathrm{~S}, \mathrm{SO}_{2}$, and $\mathrm{CS}_{2}$. The $\mathrm{S} 2 p$ ionization potentials (IPs) of $\mathrm{H}_{2} \mathrm{~S}, \mathrm{SO}_{2}$, and $\mathrm{CS}_{2}$ are $170.6[1,15]$, 175.2 [1], and $170.2 \mathrm{eV} \mathrm{[16],} \mathrm{respectively.} \mathrm{Similar} \mathrm{S} 2 p$ IPs measured by conventional electron spectroscopy for $\mathrm{H}_{2} \mathrm{~S}$ and $\mathrm{CS}_{2}$, however, do not imply similar chemical environments (i.e., screening or antiscreening in the neutral ground state due to electronegativity differences of the atoms in these two molecules). Such chemical information is masked by the orbital relaxation effect which arises from changes in the electron density distribution of valence electrons in the atom where the inner-shell hole is created. In the present work, we demonstrate that we can disentangle the relaxation effect and the intrinsic chemical effect by measuring double-ionization potentials (DIPs) of single-site DCH states using multielectron coincidence spectroscopy and the relevant theoretical description based on second-order perturbation theory.

\section{EXPERIMENTAL DETAILS}

The experiments were performed on beamline U49/2PGM-2 [17] at the synchrotron radiation storage ring BESSYII in Berlin, using a magnetic bottle time-of-flight (TOF) electron kinetic energy analyzer [18,19] capable of multiparticle coincidence detection. All $(>90 \%)$ electrons from the intersection of the soft $\mathrm{x}$-ray beam with an effusive jet of target gas were directed to a $\sim 2$-m distant microchannel plate detector by the inhomogeneous field of a conical permanent magnet and a homogeneous solenoidal guiding field. The measured detection efficiency in these experiments was around $50 \%$ and the numerical energy resolution $E / \Delta E$ for single electrons was about 50. Calibration of the conversion from electron TOF to kinetic energy was done using the known energies of Auger electrons from Xe, refined for each individual target gas, by the known $L$-shell single-ionization energies $[1,15,16]$.

\section{RESULTS AND DISCUSSION}

Spectra of the $\mathrm{S} 2 p$ DCH states in $\mathrm{H}_{2} \mathrm{~S}, \mathrm{SO}_{2}$, and $\mathrm{CS}_{2}$, recorded at the photon energy of $h v=500 \mathrm{eV}$, are shown in Fig. 1. As can be seen, all spectra show three distinct spectral lines which can be assigned to the ${ }^{3} P,{ }^{1} D$, and ${ }^{1} S$ final states, respectively. The corresponding energies are listed in Table I. For some of the species, similar spectra were obtained at other photon energies $\left(h v=550 \mathrm{eV}\right.$ for $\mathrm{H}_{2} \mathrm{~S} ; h v=480 \mathrm{eV}$ and $550 \mathrm{eV}$ for $\mathrm{SO}_{2}$ ) to confirm the observed features; they all show peaks at the same positions within a conservative 


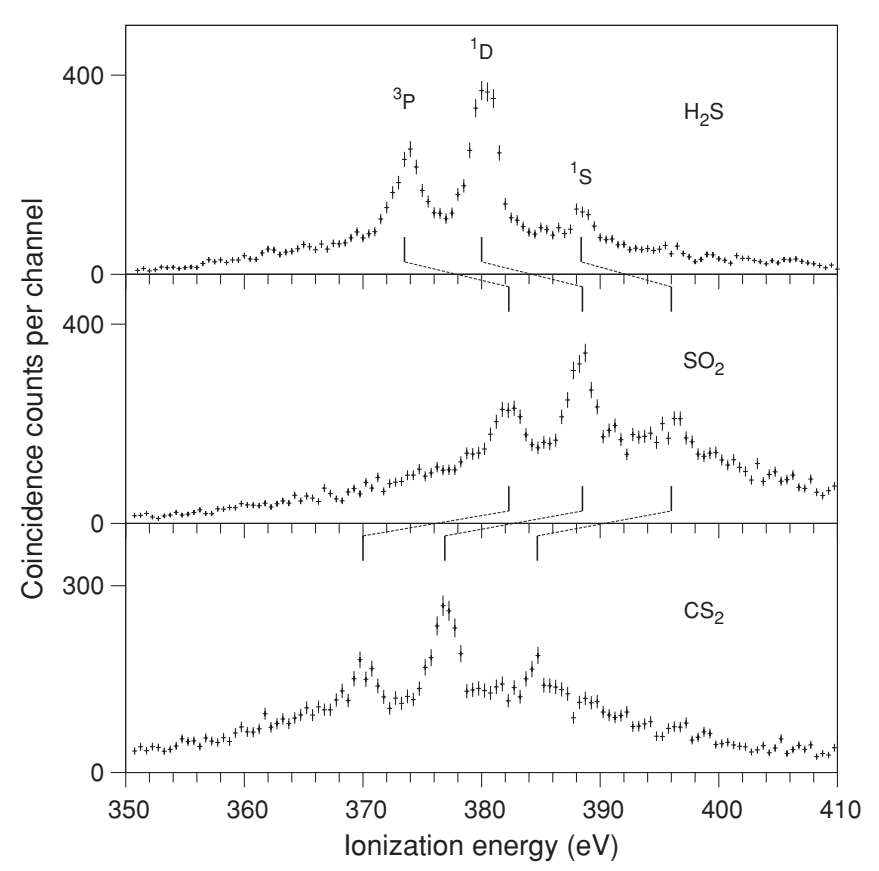

FIG. 1. S $2 p$ DCH spectra for $\mathrm{H}_{2} \mathrm{~S}, \mathrm{CS}_{2}$, and $\mathrm{SO}_{2}$ recorded at $h v=500 \mathrm{eV}$. The spectra have been extracted from triple coincidence data as described in the text and reflect for each of the species the ${ }^{3} P$, ${ }^{1} D$, and ${ }^{1} S$ final states, respectively. The corresponding ionization energies are given in Table I.

experimental uncertainty of the absolute energy positions of about $\pm 0.5 \mathrm{eV}$, which can be traced back to a systematic uncertainty in absolute electron flight-time calibration of around $1 \mathrm{~ns}$. Estimates of statistical errors of the individual line positions are given in Table I. The energies of the ${ }^{1} D$ state of $\mathrm{H}_{2} \mathrm{~S}$ and $\mathrm{SO}_{2}$ can be extracted from an early Auger study of Keski-Rahkonen et al. [20] and show good agreement with our experimental values. To the best of our knowledge, the corresponding energy value for $\mathrm{CS}_{2}$ has not been established before, nor have those of any of the other electronic states resolved here.

The spectra were extracted as electron pair energy sums from triple coincidence events akin to our very recent work [14]; here the sum of all three electron energies was constrained to lie within a range of $\pm 30 \mathrm{eV}$ from the expected position of the triply ionized states of one core and two valence holes (CVV) in DCH decay. The latter are found as the main intermediate electronic states in relaxation of the DCH states to quadruply ionized valence electronic states (cf. Ref. [14]). The details of these more highly charged states will be discussed elsewhere. In order to clarify the spectra from interfering background which comes mainly from emission of secondary electrons when the abundant Auger electrons from double ionization hit solid surfaces, we have modeled these secondary electrons as smooth distributions based on the observed low-energy electron spectra, and have subtracted the resulting background in the form of two-dimensional coincidence maps, from the raw coincidence data (cf. Ref. [14]). The final state intensities are determined from peak areas after subtraction of the residual smooth background represented as a polynomial fit.
TABLE I. Experimental (Expt.) and calculated (Theor.) DIP and IP values for the $2 p^{-2}$ and $2 p^{-1}$ states of $\mathrm{H}_{2} \mathrm{~S}, \mathrm{SO}_{2}$, and $\mathrm{CS}_{2}$, respectively (the experimental IPs are from Refs. $[1,15,16]$ ). Statistical errors for the experimental values are given in parentheses. Averaged values (Av.) are obtained according to statistical weight. $\epsilon$ denotes HF orbital energies. All energies are in $\mathrm{eV}$.

\begin{tabular}{lllllllr}
\hline \hline & & \multicolumn{2}{c}{ DIP $\left(\mathrm{S} 2 p^{-2}\right)$} & & \multicolumn{2}{c}{ IP $\left(\mathrm{S} 2 p^{-1}\right)$} \\
\cline { 3 - 4 } & & \multicolumn{1}{c}{ Expt. } & Theor. & & Expt. & Theor. \\
\hline $\mathrm{H}_{2} \mathrm{~S}$ & ${ }^{3} P$ & $373.5(0.2)$ & 373.8 & ${ }^{2} P_{3 / 2}$ & 170.2 & 170.3 \\
& ${ }^{1} D$ & $380.0(0.2)$ & 380.6 & ${ }^{2} P_{1 / 2}$ & 171.4 & 171.5 \\
& ${ }^{1} S$ & $388.4(0.2)$ & 390.5 & & & \\
& $\mathrm{Av}$. & 376.7 & 377.2 & Av. & 170.6 & 170.7 \\
& & & & & $\epsilon$ & -180.9 \\
$\mathrm{SO}_{2}$ & ${ }^{3} P$ & $382.1(0.2)$ & 383.8 & ${ }^{2} P_{3 / 2}$ & 174.8 & 175.3 \\
& ${ }^{1} D$ & $388.4(0.2)$ & 390.1 & ${ }^{2} P_{1 / 2}$ & 176.1 & 176.5 \\
& ${ }^{1} S$ & $396.1(0.4)$ & 396.4 & & & \\
& Av. & 385.1 & 386.7 & & Av. & 175.2 & 175.7 \\
& & & & $\epsilon$ & -186.4 \\
$\mathrm{CS}_{2}$ & ${ }^{3} P$ & $370.0(0.2)$ & 370.6 & ${ }^{2} P_{3 / 2}$ & 169.8 & 170.0 \\
& ${ }^{1} D$ & $376.9(0.2)$ & 377.6 & ${ }^{2} P_{1 / 2}$ & 171.1 & 171.1 \\
& ${ }^{1} S$ & $384.7(0.4)$ & 385.7 & & & \\
& Av. & 373.3 & 373.9 & Av. & 170.2 & 170.4 \\
& & & & & $\epsilon$ & -181.7 \\
\hline \hline
\end{tabular}

To validate and interpret the experimental spectra, we have carried out high-level quantum chemical calculations for all three species under investigation. The molecular geometries in these calculations were optimized at the secondorder Møller-Plesset (MP2) correlation-consistent polarized valence triple-zeta (cc-pVTZ) level of approximation using GAUSSIAN03 [21]. Multiconfigurational self-consistent field (MCSCF) calculations of the vertical IPs and DIPs of the $\mathrm{SCH}$ and DCH states, respectively, were performed to compute the relaxed ionization energies. The active space was set to all occupied and unoccupied valence orbitals except for the $1 s$ core orbitals, and double excitation configurations to the unoccupied orbitals were included in the configurations. In order to include the $2 p$ core orbital relaxation effects properly, the following two alternative MCSCF calculations were iterated several times: The $2 p$ core orbitals were fixed in one of the MCSCF calculations and the other orbitals in the active space were fixed in the other calculations. Orbital exponents of the triply augmented (aug) cc-pVTZ basis set were used for the core-hole sulfur atom without any contractions, whereas contracted aug-cc-pVTZ basis sets were used for the other atoms. For $\mathrm{CS}_{2}$, an effective core potential for the non-core-hole sulfur atom was applied to avoid difficult convergence. To check the basis-set dependence of our numerical results, aug-cc-pVQZ basis sets were also used for $\mathrm{H}_{2} \mathrm{~S}$. All MCSCF calculations were performed using the MOLPRO2009 program [22].

In Table I the measured DIPs for the creation of the $\mathrm{S} 2 p$ double inner shell holes in $\mathrm{H}_{2} \mathrm{~S}, \mathrm{SO}_{2}$, and $\mathrm{CS}_{2}$ are compared with our numerical MCSCF results. We note that the $\mathrm{S} 2 p$ IPs and DIPs for $\mathrm{H}_{2} \mathrm{~S}$ have been investigated before by Ågren et al. [6] using an ab initio method, and our theoretical results are consistent with their results. As can be seen from Table I, 
the agreement between the experimental and theoretical values is sufficiently good.

In looking at both the experimental and theoretical energy spacings between the ${ }^{3} P,{ }^{1} D$, and ${ }^{1} S$ states, we note that these values are nearly constant across the molecules. We can associate this finding primarily with the exchange interaction that reflects the atomic inner-shell nature of $\mathrm{S} 2 p$. The repulsion-exchange energies of these three states can be approximated as

$$
\begin{aligned}
& { }^{3} P: V_{a b a b}-V_{a b b a}, \\
& { }^{1} D: V_{a b a b}+V_{a b b a}, \\
& { }^{1} S: V_{a b a b}+4 V_{a b b a},
\end{aligned}
$$

where $V_{a b a b}=\langle a b \mid a b\rangle$ and $V_{a b b a}=\langle a b \mid b a\rangle$ are the Coulomb repulsion and exchange integrals, respectively, and $a$ and $b$ are two different components of $2 p$. Both the experimental and theoretical energy spacings between ${ }^{3} P$ and ${ }^{1} D$ agree reasonably with the calculated value of $2 V_{a b b a}$, whereas those between ${ }^{1} D$ and ${ }^{1} S$ are systematically smaller than $3 V_{a b b a}$, indicating electron correlation effects. For later use, we introduce here the approximate average state energies of $2 p^{-2}$ DIP (denoted as Av. in Table I), where both the experimental and theoretical energies for the ${ }^{3} P,{ }^{1} D$, and ${ }^{1} S$ states are weighted according to their statistical ratio of $9: 5: 1$. In this way we can average out the exchange energies $V_{a b b a}$ among the three states and approximate the averaged hole-hole repulsion energy to a single value $V_{a b a b}$. It should be noted that the experimentally observed intensities do not reflect the statistical weights, and we shall come back to this point further below.

Table I also contains experimental and theoretical IP values of the corresponding single inner-shell-hole creation $[1,15,16]$. The experimental and theoretical results are in good agreement, as seen in this table. For later use, we also obtained statistically weighted average state energies of the spin-orbit split ${ }^{2} P_{3 / 2}$ and ${ }^{2} P_{1 / 2}$ components.

The energy differences

$$
\Delta E=\operatorname{DIP}\left(\mathrm{S} 2 p^{-2}\right)-2 \operatorname{IP}\left(\mathrm{S} 2 p^{-1}\right),
$$

for the averaged experimental and theoretical DIPs and IPs are given in Table II. According to Ref. [8], a single $2 p$ hole IP of a molecule can be approximated as

$$
\mathrm{IP}=-\epsilon\left(\mathrm{S} 2 p^{-1}\right)-\mathrm{RC}\left(\mathrm{S} 2 p^{-1}\right),
$$

where $\epsilon\left(\mathrm{S} 2 p^{-1}\right)$ is the Hartree-Fock (HF) orbital energy of the neutral molecule, as given in Table I, and $\mathrm{RC}\left(\mathrm{S} 2 p^{-1}\right)$ is the generalized relaxation energy that includes both orbital relaxation energy and correlation energy. Analogously, an inner-shell DIP (averaged value) of the molecule at the same atomic site can be approximated as

$$
\mathrm{DIP}=-2 \epsilon\left(\mathrm{S} 2 p^{-1}\right)-2 \mathrm{RC}\left(\mathrm{S} 2 p^{-1}\right)-\operatorname{ERC}\left(\mathrm{S} 2 p^{-2}\right)+V_{a b a b}
$$

Here the averaged single-site two-hole repulsion integral $V_{a b a b}$ is calculated to be $54.5 \mathrm{eV}$ for all three molecules studied. $\operatorname{ERC}\left(\mathrm{S} 2 p^{-2}\right)$ is the generalized excess relaxation energy stemming from nonadditive contributions to the double-core-

\begin{tabular}{|c|c|c|c|c|c|}
\hline & \multicolumn{2}{|c|}{$\Delta E$} & \multicolumn{2}{|c|}{$\mathrm{ERC} / 2$} & \multirow{2}{*}{$\begin{array}{c}\mathrm{R} \\
\text { Theor. }\end{array}$} \\
\hline & Expt. & Theor. & Expt. & Theor. & \\
\hline $\mathrm{H}_{2} \mathrm{~S}$ & 35.5 & 35.8 & 9.5 & 9.4 & 10.1 \\
\hline $\mathrm{SO}_{2}$ & 34.7 & 35.3 & 9.9 & 9.6 & 9.8 \\
\hline $\mathrm{CS}_{2}$ & 32.9 & 33.1 & 10.8 & 10.7 & 11.2 \\
\hline
\end{tabular}
hole creation. In Table II we present the corresponding values for $\operatorname{ERC}\left(\mathrm{S} 2 p^{-2}\right) / 2$. According to second-order perturbation
TABLE II. The energy difference $\Delta E$ [cf. Eq. (1)], half of the excess energy ERC/2 [cf. Eq. (3)] and the strict relaxation energy $\mathrm{R}$ for $\mathrm{H}_{2} \mathrm{~S}, \mathrm{SO}_{2}$, and $\mathrm{CS}_{2}$. All energies are in $\mathrm{eV}$.

theory, $\mathrm{ERC}\left(\mathrm{S} 2 p^{-2}\right)$ can be approximated as twice the $\mathrm{SCH}$ relaxation energy $\mathrm{R}\left(\mathrm{S} 2 p^{-1}\right)$ [8]. The difference between the $\triangle \mathrm{SCF}$ calculation for IP and the binding energy $\epsilon\left(\mathrm{S} 2 p^{-1}\right)$ obtained by the HF calculation gives the strict relaxation energy $\mathrm{R}\left(\mathrm{S} 2 p^{-1}\right)$ for which this relation holds. We thus compare the values of $\operatorname{ERC}\left(\mathrm{S} 2 p^{-2}\right) / 2$ extracted from the experiments and the MCSCF calculations with $a b$ initio values of $\mathrm{R}\left(\mathrm{S} 2 p^{-1}\right)$ thus obtained. A reasonable agreement confirms the validity of the present treatment.

In comparing the results given in Tables I and II, we find that, although the IPs are nearly the same for $\mathrm{H}_{2} \mathrm{~S}$ and $\mathrm{CS}_{2}$, their DIPs and their relaxation energies $\mathrm{R}\left(\mathrm{S} 2 p^{-1}\right)$ are significantly different. The relaxation energy is, according to both the experimental and theoretical results, apparently largest for $\mathrm{CS}_{2}$ among the three molecular systems investigated here. Such a difference can be explained as follows: The relaxation energy is mainly associated with changes in the electron density distribution of the valence electrons in the atom where the inner-shell holes are created. In the case of $\mathrm{CS}_{2}$, where the electron density flows efficiently from the CS moiety to the $\mathrm{S}$ atom with a $2 p$ hole, the relaxation energy is noticeably larger than that of $\mathrm{H}_{2} \mathrm{~S}$, where the electron density has already been drawn to the sulfur atom in the ground state where there are very few electrons that can participate in the orbital relaxation. As a result, the DIPs for these two molecules are significantly different in spite of nearly identical IP values.

The fact that we can extract the relaxation energy $\mathrm{R}\left(\mathrm{S} 2 p^{-1}\right)$ $\left[\sim \operatorname{ERC}\left(\mathrm{S} 2 p^{-2}\right) / 2\right]$ from the present DIP measurements has further implications; namely, we can in principle extract the intrinsic chemical information hidden in the conventional IP measurements. Our experimental finding of a larger relaxation energy in $\mathrm{CS}_{2}$ than in $\mathrm{H}_{2} \mathrm{~S}$, in contrast to the fact that the two molecules have approximately the same IP, may be rationalized by the stronger antiscreening effect in the $\mathrm{CS}_{2}$ molecule. This conclusion may also be extracted from the $2 p$ orbital energy of $\mathrm{CS}_{2}$ at the HF level calculation, which is larger than that of $\mathrm{H}_{2} \mathrm{~S}$. However, this chemical information (i.e., the screening or antiscreening in the neutral ground state due to electronegativity differences of the respective atoms) is superimposed upon the relaxation effect in conventional core-level photoelectron spectroscopy and has been hitherto experimentally hidden.

Let us turn our attention once more to Fig. 1. As pointed out above, the line intensity pattern is very different from the statistical weights, in particular for the ${ }^{3} P:{ }^{1} D$ ratio, where the intensity of ${ }^{3} P$ is much lower than that of ${ }^{1} D$. This is observed for all three molecules investigated here, and stands in clear contrast to what is known from $\mathrm{S} 2 p \mathrm{SCH}$ 
formation of these systems $[1,15,16]$. A similar discrepancy has been noted recently by Kaneyasu et al. [23] for valence double ionization of atomic Ne $2 p$ orbitals. The final DCH states, or more precisely the corresponding double $(2 h-2 p)$ excitations with 2 excited electrons in the continuum, acquire intensity not directly, but only via the small admixtures of single $(h-p)$ excitations, which will be heavily state dependent. Thus, one should not expect the intensities to simply reflect the statistical weights of the final dicationic states. Quantitative understanding for the origin of this nonstatistical intensity distribution requires further theoretical investigations.

\section{CONCLUSIONS}

In summary, by examining the $\mathrm{S} 2 p^{-2} \mathrm{DCH}$ states of $\mathrm{H}_{2} \mathrm{~S}^{2+}, \mathrm{SO}_{2}{ }^{2+}$ and $\mathrm{CS}_{2}{ }^{2+}$ using a multielectron coincidence technique, we have demonstrated that DCH spectroscopy can be used to separate the intrinsic chemical effect from the orbital relaxation effect that often masks the interesting chemical information. The present findings illustrate nicely the power of DCH spectroscopy for chemical analysis that can be readily used for many other small and larger molecular systems in both gas and condensed phases.

\section{ACKNOWLEDGMENTS}

This work has been financially supported by the Swedish Research Council (VR), the Göran Gustafsson Foundation (UU/KTH), and the Knut and Alice Wallenberg Foundation, Sweden. This work was also supported by the European Community-Research Infrastructure Action under the FP6 "Structuring the European Research Area" Programme (through the Integrated Infrastructure Initiative "Integrating Activity on Synchrotron and Free Electr-on Laser Science"Contract R II 3-CT-2004-506008). K.U. acknowledges the support for the X-ray Free Electron Laser Utilization Research Project of Ministry of Education, Culture, Sports, Science and Technology of Japan. We are grateful to N. V. Kryzhevoi for sending us some values of the two-electron integrals, and L. S. Cederbaum, M. Ehara, S. Yabushita, and S. Fritzsche for stimulating discussions.
[1] K. Siegbahn et al., ESCA Applied to Free Molecules (NorthHolland, Amsterdam, 1969).

[2] L. S. Cederbaum, F. Tarantelli, A. Sgamellotti, and J. Schirmer, J. Chem. Phys. 85, 6513 (1986).

[3] L. S. Cederbaum, Phys. Rev. A 35, 622 (1987).

[4] L. S. Cederbaum, F. Tarantelli, A. Sgamellotti, and J. Schirmer, J. Chem. Phys. 86, 2168 (1987).

[5] E. M.-L. Ohrendorf, F. Tarantelli, and L. S. Cederbaum, Chem. Phys. Lett. 183, 1 (1991).

[6] H. Å. Gren and H. J. Aa, Chem. Phys. 172, 45 (1993).

[7] R. Santra, N. V. Kryzhevoi, and L. S. Cederbaum, Phys. Rev. Lett. 103, 013002 (2009).

[8] M. Tashiro, M. Ehara, H. Fukuzawa, K. Ueda, C. Buth, N. V. Kryzhevoi, and L. S. Cederbaum, J. Chem. Phys. 132, 184302 (2010).

[9] M. Tashiro, M. Ehara, and K. Ueda, Chem. Phys. Lett. 496, 217 (2010).

[10] M. Hoener, L. Fang, O. Kornilov, O. Gessner, S. T. Pratt, M. Gühr, E. P. Kanter, C. Blaga, C. Bostedt, J. D. Bozek, P. H. Bucksbaum, C. Buth, M. Chen, R. Coffee, J. Cryan, L. DiMauro, M. Glownia, E. Hosler, E. Kukk, S. R. Leone, B. McFarland, M. Messerschmidt, B. Murphy, V. Petrovic, D. Rolles, and N. Berrah, Phys. Rev. Lett. 104, 253002 (2010).

[11] L. Young, E. P. Kanter, B. Krässig, Y. Li, A. M. March, S. T. Pratt, R. Santra, S. H. Southworth, N. Rohringer, L. F. DiMauro, G. Doumy, C. A. Roedig, N. Berrah, L. Fang, M. Hoener, P. H. Bucksbaum, J. P. Cryan, S. Ghimire, J. M. Glownia, D. A. Reis, J. D. Bozek, C. Bostedt, and M. Messerschmidt, Nature (London) 466, 56 (2010).

[12] L. Fang, M. Hoener, O. Gessner, F. Tarantelli, S. T. Pratt, O. Kornilov, C. Buth, M. Gühr, E. P. Kanter, C. Bostedt, J. D. Bozek, P. H. Bucksbaum, M. Chen, R. Coffee, J. Cryan,
M. Glownia, E. Kukk, S. R. Leone, and N. Berrah, Phys. Rev. Lett. 105, 083005 (2010).

[13] [http://lcls.slac.stanford.edu/]

[14] J. H. D. Eland, M. Tashiro, P. Linusson, M. Ehara, K. Ueda, and R. Feifel, Phys. Rev. Lett. 105, 213005 (2010).

[15] R. G. Cave and K. H. Tan, Chem. Phys. Lett. 197, 161 (1992).

[16] H. Wang, M. Bässler, I. Hjelte, F Burmeister, and L. Karlsson, J. Phys. B 34, 1745 (2001).

[17] D. R. Batchelor, R. Follathb, and D. Schmeißer, Nucl. Instrum. Methods A 467, 470 (2001).

[18] J. H. D. Eland, O. Vieuxmaire, and T. Kinugawa, Phys. Rev. Lett. 90, 053003 (2003).

[19] J. H. D. Eland, P. Linusson, L. Hedin, E. Andersson, J.-E. Rubensson, and R. Feifel, Chem. Phys. Lett. 485, 21 (2010).

[20] O. Keski-Rahkonen and M. O. Krause, J. Electron Spectrosc. Relat. Phenom. 9, 371 (1976).

[21] M. J. Frisch et al., Gaussian 03, Revision C.02 (Gaussian, Inc., Wallingford CT, 2004).

[22] MOLPRO, version 2009.1, a package of ab initio programs, H.-J. Werner, P. J. Knowles, F. R. Manby, M. Schütz, P. Celani, G. Knizia, T. Korona, R. Lindh, A. Mitrushenkov, G. Rauhut, T. B. Adler, R. D. Amos, A. Bernhardsson, A. Berning, D. L. Cooper, M. J. O. Deegan, A. J. Dobbyn, F. Eckert, E. Goll, C. Hampel, A. Hesselmann, G. Hetzer, T. Hrenar, G. Jansen, C. Köppl, Y. Liu, A. W. Lloyd, R. A. Mata, A. J. May, S. J. McNicholas, W. Meyer, M. E. Mura, A. Nicklaß, P. Palmieri, K. Pflüger, R. Pitzer, M. Reiher, T. Shiozaki, H. Stoll, A. J. Stone, R. Tarroni, T. Thorsteinsson, M. Wang, A. Wolf, see [http://www.molpro.net].

[23] T. Kaneyasu, Y. Hikosaka, E. Shigemasa, F. Penent, P. Lablanquie, T. Aoto, and K. Ito, Phys. Rev. A 76, 012717 (2007). 